\title{
Clinical Application and Research Progress of Accelerated Rehabilitation Surgery in Perioperative Period of Advanced Gastric Cancer in the Elderly
}

\author{
Chengpeng Ran, Guangwei Gong* \\ Wuhan University of Science and Technology Affiliated Xiaogan Hospital, Hubei Xiaogan, China \\ Email:2292988066@qq.com, *2054530895@qq.com
}

How to cite this paper: Ran, C.P. and Gong, G.W. (2020) Clinical Application and Research Progress of Accelerated Rehabilitation Surgery in Perioperative Period of Advanced Gastric Cancer in the Elderly. International Journal of Clinical Medicine, 11, 101-110.

https://doi.org/10.4236/ijcm.2020.113010

Received: January 17, 2020

Accepted: February 29, 2020

Published: March 3, 2020

Copyright $\odot 2020$ by author(s) and Scientific Research Publishing Inc. This work is licensed under the Creative Commons Attribution International License (CC BY 4.0).

http://creativecommons.org/licenses/by/4.0/

\begin{abstract}
Enhanced recovery after surgery (ERAS) has been used in various surgical professions in recent years and is widely accepted by doctors. This concept not only helps patients speed up postoperative recovery, reduce the incidence of related complications and shorten hospital stays, but also has been proved to be effective and safe in the perioperative application of gastric cancer. This article reviews the clinical application and research progress of enhanced recovery after surgery in the perioperative period of advanced gastric cancer in the elderly.
\end{abstract}

\section{Keywords}

Enhanced Recovery after Surgery, Elderly Patients, Advanced Gastric Cancer, Perioperative Period, Clinical Application, Research Progress

\section{Overview}

According to GLOBOCAN data in 2018 [1] [2], the number of gastric cancer patients worldwide is about 103 million. Gastric cancer has become the fifth most common malignant tumor and one of the third most deadly tumors. New cases of gastric cancer are added every year. About $5.7 \%$ of all new cancer cases. In China, although the incidence and mortality of gastric cancer have declined in recent years, it accounted for about 11 percent of all cancers and 12.8 percent of all deaths. It is still the third leading cause of cancer death in China, after lung cancer and colorectal cancer. In the elderly, the incidence of gastric cancer is *Corresponding author. 
higher [3], because the clinical symptoms of early gastric cancer are not obvious, patients often do not pay enough attention. In addition, due to the lack of effective methods for early screening of gastric cancer, the detection rate of early gastric cancer is only about $10 \%$ [4]. Has entered the advanced stage, so surgery is currently recognized as the best treatment.

In China, in recent years, the population structure tends to be aging, the proportion of the elderly ( $>60$ years old) is increasing, and the corresponding elderly patients are gradually increasing. As the body immunity of the elderly declines, the adaptability to the internal and external environment decreases. Often combined with chronic diseases, such as hypertension, diabetes, heart disease, etc. [5], the tolerance and resilience to surgery and its postoperative decline, prone to a series of complications, and even life-threatening. How to reduce stress response and speed up postoperative recovery is a hot and difficult point.

Based on evidence-based medicine, ERAS is a multidisciplinary collaboration that reduces post-stress and accelerates postoperative recovery. It was first proposed by Danish surgeon Kehlet et al. [6] in 1997 and in China by 2007. Professor Zhiwei's application in gastric cancer [7] has been widely used in various surgical fields. This concept runs through preoperative, intraoperative and postoperative operations. The focus is on preoperative consultation, optimized nutrition, standardized analgesia, and reduction. The use of opioids to minimize electrolyte and fluid imbalances and promote early activity and feeding [8]; there is clear evidence [9] [10] that accelerating rehabilitation surgery is safe and effective for elderly patients, supporting accelerated surgical surgery applications. In elderly patients, this article reviews the clinical application and research progress of accelerated rehabilitation surgery in elderly patients with advanced gastric cancer during gastric surgery.

\section{Application of Eras in Perioperative Period of Advanced Gastric Cancer in the Elderly}

\subsection{Preoperative Preparation}

\subsubsection{Preoperative Communication and Consultation}

Before surgery, the patient physiologically responds to stress. The central nervous system mediates this process, producing stress hormones and inflammatory mediators [11], and strengthens effective communication with patients before surgery. It can be written and spoken. The above agreement provides individualized counseling to patients and psychologically prepares them for what they are doing, thereby reducing the anxiety and fear of patients, improving recovery, improving patient compliance with the plan, and promoting eating. Early exercise, pain control and respiratory function, reduce the occurrence of complications [8] [12]; for elderly patients, often combined with chronic diseases, to actively treat diseases that affect the recovery of patients, such as hypertension, diabetes, blood pressure Achieve a stable state with blood glucose before surgery, and actively improve nutrition for malnourished patients, so that the patient's 
body reaches an optimal state before surgery.

\subsubsection{Preoperative Nutritional Improvement}

For elderly patients with gastric cancer, often combined with malnutrition, is the main clinical problem of gastrointestinal malignant tumors, malnutrition in hospitalized patients reached $50 \%-60 \%$, up to $71 \%$ in cancer patients [13]; malnutrition led to hospitalization Prolonged time, increased postoperative complications, slow recovery of postoperative bowel function, high readmission rate, high postoperative mortality; evidence [14], preoperative nutritional status is a key determinant of optimal outcome in gastrointestinal surgery avoid preoperative fasting before surgery, optimize preoperative nutrition, oral nutritional supplements and immunonutrient preparations; Sonvi research shows [15], preoperative oral immunonutrition preparations, postoperative complications are lower, it is recommended that all preoperative The patient underwent 5 to 7 days of on-site immunonutrition [16].

\subsubsection{Gastrointestinal Preparation}

Inadequate mechanical bowel preparation before surgery has been shown to cause dehydration and imbalance of fluids and electrolytes, especially in elderly patients. Mechanical bowel preparation is to remove solid excretion from the large intestine and reduce the amount of bacteria. Liquefaction of feces increases the risk of surgical spillage [8]; there is no need to fast for one night before surgery. The current guidelines recommended by the American Society of Anesthesiologists [17], fasting solid foods 8 hours before surgery, 2 hours before the ban According to the Cochrane study [18], the risk and incidence of inhalation were not increased compared with traditional requirements; prolonged fasting $(>12 \mathrm{~h})$ resulted in hypovolemia, increased metabolic stress and insulin resistance, insulin resistance It is a key factor for long-term rehabilitation and increased morbidity; a data suggest that [19], decreased insulin sensitivity, will lead to increased complication rate, postoperative hyperglycemia, increased risk of postoperative infection by 30\%; 2 - 3 day before surgery Drinking $300-400 \mathrm{ml}$ of carbohydrates a day can not only reduce pre-operative thirst, hunger, anxiety and postoperative insulin resistance, but also reduce postoperative nitrogen and protein loss, better maintain capacity and muscle mass, and exhaust time. With hospitalizationShortening, however, for patients with diabetes and delayed gastric emptying, gastrointestinal motility disorders with the exception of patients [8].

\subsubsection{Gastrointestinal Decompression Tube}

The nasogastric tube is not routinely used. According to the Cochrane conclusion [18], the nasogastric tube is not routinely used, the pulmonary complications are significantly reduced, the early feeding is improved, and the hospital stay is shortened. The use of the nasogastric tube has a certain effect on the nasopharyngeal mucosa. The stimulation, which leads to increased complications in the pharynx and lungs, also exerts pressure on the patient's psychology, and 
also has an effect on the patient's early bed-out activities; a randomized trial [20], no patients who received the nasogastric tube, there was no difference in postoperative nausea, vomiting, and recovery of bowel function.

\subsection{Intraoperative Application}

\subsubsection{Intraoperative Anesthesia}

The anesthesia program will promote the recovery of gastrointestinal motility and is the key to speeding up recovery. For elderly patients, the anesthesia should be avoided too deeply, and the depth of anesthesia is bis $<45$, not only to minimize the anesthetic, but also to prevent convulsions, promote rapid arousal and recovery [21]; anesthesia is a stress response that limits surgery to fluid balance and pain [22]; intraoperative intravenous anesthesia combined with epidural anesthesia, anesthesia induction and continuous anesthesia are generally selected for short-acting anesthetics (propofol, cis Lacurium, fentanyl, to reduce sympathetic nerve stimulation and postoperative anesthesia, combined anesthesia combined with general anesthesia onset, intraoperative anesthesia and epidural anesthesia can promote postoperative gastrointestinal function recovery, postoperative analgesia The advantages can not only achieve the desired anesthetic effect, but also accelerate the recovery of patients after surgery, inhibit the occurrence of postoperative intestinal paralysis, accelerate the recovery of gastrointestinal function, and provide conditions for early postoperative feeding [21].

\subsubsection{The Use of Preventive Antibiotics}

The use of prophylactic antibiotics can reduce the incidence of postoperative infections; studies have shown [23] that prophylactic antibiotics are effective against possible anaerobic and aerobic infections, 30 minutes to 1 hour before skin incision Dosage can be administered repeatedly according to the half-life of the drug and the operation time during the operation.

\subsubsection{Surgical Operation}

The safety and efficacy of laparoscopic radical gastrectomy have been confirmed [24]; there is evidence [14] that laparoscopic surgery can reduce trauma, reduce intraoperative blood loss, and reduce surgical stress response compared with conventional laparotomy Postoperative pain and discomfort, postoperative intestinal adhesions, low incidence of intestinal obstruction, early recovery of bowel function, reduced hospital stay, and a high degree of synergy with eras.

\subsubsection{Liquid Management}

Liquid management is an important part of eras. It can maintain normal blood volume by pre-operative, intraoperative and postoperative methods. Whether it is a large amount of infusion or a limited amount of infusion, it can significantly impair organ function and will increase postoperative complications $(\mathrm{P}<0.05)$. Symptoms and prolonged hospital stay [25]; there is evidence [8], a large number of fluid replacement, delayed recovery of gastrointestinal function, wound 
healing and anastomotic healing, affecting tissue oxygenation, leading to prolonged hospital stay; intraoperative fluid management is maintained There is sufficient circulation at the end of the organ, low blood volume leads to hypoperfusion, increased risk of organ failure, high blood volume leads to peripheral and pulmonary edema, and the incidence of postoperative intestinal obstruction increases [18]; intraoperative fluid should be zero-balanced For the purpose of high-risk patients or large-scale abdominal surgery should be targeted to use liquid therapy, using objective parameters, according to hemodynamics, such as cardiac output, pulse pressure changes, etc., to make patients' fluid recovery more accurate, reduce postoperative bowel Obstruction occurs, promote the recovery of bowel function, thereby reducing hospital stay [26]; postoperative, early oral fluid, limiting postoperative intravenous rehydration, can reduce hospital stay, reduce concurrency, Such as the occurrence of intestinal obstruction [8].

\subsubsection{Intraoperative Temperature}

In gastric cancer surgery, due to anesthesia leading to abnormal body temperature regulation, a large number of infusions, and prolonged exposure to the wound, cold water flushing the abdominal cavity, often lead to hypothermia, especially in elderly patients [7]; According to studies [8], maintaining a normal body temperature can reduce wound infections and heart complications. bleeding and blood transfusion requirements, shorten postoperative recovery time; it is recommended to use room temperature at $24^{\circ} \mathrm{C}$, through the upper body forced air heating, insulation blanket, heated intravenous infusion, warm water to wash the abdominal cavity and other measures to make the patient's body temperature Keep at around $36^{\circ} \mathrm{C}$ to avoid complications and improve intraoperative and postoperative safety [27].

\subsubsection{Drainage Tube}

Abdominal drainage tube should not be used routinely. Abdominal drainage tube does not reduce the incidence of complications, but increases the risk of infection and postoperative fistula [28]; a rct experiment shows [29], two groups in wound infection and there was no significant difference in postoperative pus. The incidence of postoperative complications and hospitalization time were lower in the non-drainage group; if it was in some special cases, such as anastomotic blood supply, cancerous ascites, weak constitution, nutritional status If the difference is poor, the drainage tube should be placed according to the intraoperative condition. The amount and nature of the drainage fluid should be closely observed after operation, and the tube should be extubated as soon as possible to reduce the tube stimulation and complications.

\section{Postoperative Application}

\subsection{Multimodal Analgesia}

Postoperative multimodal analgesia, preventive analgesia, the purpose of early 
mobilization, promote the recovery of gastrointestinal function; multimodal analgesia is the use of more than one pain control mode to achieve effective analgesia, including systemic administration, Regional, epidural analgesia, axonal pattern, and a variety of drugs designed to reduce the use of opioids and reduce the side effects caused by opioids; opioids cause intestinal obstruction, respiratory depression, causing nausea, vomiting, especially in the elderly Large doses of opioids increase the risk [30]; multimodal analgesia also incorporates prophylactic analgesia, which is the treatment of pre-drugs that reduce pain or pain before surgery. It has been shown to reduce pain, inflammation, nausea, vomiting [31]; effective pain control is the basis for early bed-out activities, gastrointestinal recovery, and multi-mode combined analgesia is effective, not only reduces the occurrence of related complications, but also promotes early stage Bed activity, shortened hospital stay.

\subsection{Early Diet}

Early $(<24 \mathrm{~h})$ diet accelerated recovery of gastrointestinal function, reduced complication rate and mortality, and contributed to wound healing and anastomotic strength, which has been shown to be safe [16] [32]; 6 - $24 \mathrm{~h}$ began to take a small amount of warm boiled water, if the patient does not have discomfort such as bloating, the next day after surgery, gradually overdue from a fluid diet to a normal diet; early return to a normal diet, that is, provide the corresponding energy and protein, and can reduce the cause of hunger Insulin resistance [8]; according to Carr et al. [18], intestinal mucosal permeability was not increased in patients receiving early feeding; RCT experiments performed according to EINakeeb et al. [33], early diet promotes intestinal function Recover, reduce intestinal edema, shorten the time of exhaust and stool, reduce the risk of various forms of infection, thereby shortening the length of hospital stay, and recommend early diet.

\subsection{Prevention of Nausea and Vomiting}

$25 \%$ - 35\% of patients have postoperative nausea and vomiting. By administering prophylactic antiemetic drugs, $40 \%$ of postoperative nausea and vomiting can be reduced. Patients should be advised to give preventive antiemetic drugs [12] in advance; prevention of nausea and vomiting More meaningful than analgesia, reduce the risk of anastomotic leakage, promote early feeding, early bed-out activities; prevent postoperative nausea and vomiting, can be given dexamethasone sodium phosphate at the beginning of surgery or serotonin antagonism at the end of surgery Agent [8].

\subsection{Venous Thrombosis Prevention}

A common complication of venous thromboembolism, a higher incidence in elderly patients, but effective prevention strategies, including pharmacological or non-pharmacological aspects, can significantly reduce the incidence; for patients 
with epidural analgesia, should Careful use of antithrombotic drugs, before the use of low molecular weight heparin, needle should be introduced, the catheter should be taken at the lowest point of drug action (before the next scheduled dose), the subsequent dose should be given at least 2 hours after extubation, for Patients with a body mass index ( $>30$ ) should consider using higher doses of low molecular weight heparin [33].

\subsection{Catheter}

The urinary catheter should be pulled out as soon as possible after the operation, and pulled out on the first or second day after surgery to promote the patient to get out of bed early after surgery, without increasing the incidence of urinary tract infection, but pay attention to patients with benign prostatic hyperplasia, uterus Patients with a history of resection and cesarean section need to strengthen the frequency of observation after surgery. When patients have urinary dysfunction, they should be treated in time [6].

\subsection{Get out of Bed Early}

Long-term bed rest can lead to atelectasis, insulin resistance, thromboembolism, decreased exercise capacity, and in the case of effective analgesia, it is possible to get out of bed early; it is recommended to start appropriate activities the next day after surgery. It not only reduces related complications, but also promotes intestinal function recovery, strengthens blood circulation, promotes wound healing, and shortens hospital stay [12].

\section{Summary and Outlook}

The clinical application of accelerated rehabilitation surgery in perioperative period of elderly gastric cancer has been proved to be safe and effective, reducing stress response and postoperative complications, improving postoperative nutritional status, speeding up recovery, shortening hospital stay and reducing costs. However, there is still little evidence on how eras can benefit patients in the long term [8]; for the application of accelerated rehabilitation surgery, it is not possible to generalize the application of individualized conditions; the current clinical application of accelerated rehabilitation surgery still exists. The challenge is that the surgeon is accustomed to the traditional concept and lacks understanding of the eras. Second, the lack of effective communication between the doctor and the patient leads to insufficient compliance and compliance. The third is to accelerate the lack of standardization in rehabilitation surgery, and some aspects are still controversial. Lack of evidence to enable surgeons to obtain effective guidance in application; fourth, accelerating rehabilitation surgery is a multi-disciplinary collaboration, including anesthesia, nursing, etc., which leads to difficulties in application; I believe that the concept of accelerated rehabilitation surgery will continue to improve in the future. And development, widely used to benefit patients. 


\section{Conflicts of Interest}

The authors declare no conflicts of interest regarding the publication of this paper.

\section{References}

[1] Bray, F., Ferlay, J., Soerjomataram, I., et al. (2018) Global Cancer Statistics 2018: GLOBOCAN Estimates of Incidence and Mortality Worldwide for 36 Cancers Inu 185 Countries. CA: A Cancer Journal for Clinicians, 68, 394-424. https://doi.org/10.3322/caac.21492

[2] Rawlai, P. and Barsok, A. (2019) Epidemiology of Gastric Cancer: Global Trends, Risk Factors and Prevention. Gastroenterology Review, 1, 26-38. https://doi.org/10.5114/pg.2018.80001

[3] Chen, W., Zheng, R., Baade, P.D., et al. (2016) Cancer Statistics in China, 2015. CA: A Cancer Journal for Clinicians, 6, 115-132. https://doi.org/10.3322/caac.21338

[4] Chang, M., Zhang, J.C., Zhou, Q., et al. (2017) Advances in Epidemiology of Gastric Cancer. Journal of Gastroenterology and Hepatology, 26, 966-969.

[5] Shi, X.L. (2017) Foreword Application of Accelerated Rehabilitation Surgery in Elderly Patients. Practical Geriatrics, 31, 1101-1102.

[6] Mortensen, K., Nilsson, M., Slim, K., et al. (2014) Consensus Guidelines for Enhanced Recovery after Gastrectomy. British Journal of Surgery, 101, 1209-1229. https://doi.org/10.1002/bjs.9582

[7] Lu, R.Z., Lei, X., Gao, Y.T., et al. (2017) Application Progress of Accelerated Rehabilitation Surgery Concept in Gastric Cancer during Perioperative Period. World $s$ Latest Medical Information Abstracts, 17, 49-50.

[8] Michal, P., Judene, M., Jan, W., et al. (2018) Current Status of Enhanced Recover after Surgery (ERAS) Protocol in Gastrointestinal Surgery. Medical Oncology, 35, 1-8. https://doi.org/10.1007/s12032-018-1153-0

[9] Olle, L. and Martin, H. (2018) Enhanced Recovery after Surgery-ERAS-Principles, Practice and Feasibility in the Elderly. Aging Clinical and Experimental Research, 30, 249-252.

[10] Alison, L., Michael, J., Solomon, M.J. and Harrison, J.D. (2014) A Qualitative Study Assessing the Barriers to Implementation of Enhanced Recovery After Surgery. World Journal of Surgery, 38, 1374-1380. https://doi.org/10.1007/s00268-013-2441-7

[11] Ljungqvist, O., Scott, M., et al. (2017) Enhanced Recovery after Surgery: A Review. JAMA Surgery, 152, 292-298. https://doi.org/10.1001/jamasurg.2016.4952

[12] Amir, E., Ihan, E., Amjad, E., et al. (2019) Essential Elements for Enhanced Recovery after Intra-Abdominal Surgery. Current Pain and Headache Reports, 23, Article No. 21. https://doi.org/10.1007/s11916-019-0753-5

[13] Lim, S.L., Ong, K.C., Chan, Y.H., et al. (2012) Malnutrition and Its Impact on Cost of Hospitalization, Length of Stay, Readmission and 3-Year Mortality. Clinical Nutrition, 31, 345-350. https://doi.org/10.1016/j.clnu.2011.11.001

[14] Sergio, S., Geerard, B., Marco, B., et al. (2018) Perioperative Nutrition and Enhanced Recovery after Surgery in Gastrointestinal Cancer Patients. A Position Paper by the ESSO Task Force in Collaboration with the ERAS Society (ERAS Coalition). European Journal of Surgical Oncology, 44, 509-514. https://doi.org/10.1016/j.ejso.2017.12.010 
[15] Moya, P., Soriano-Irigaray, L., Ramirez, J.M., et al. (2016) Perioperative Standard Oral Nutrition Supplements versus Immunonutrition in Patients Undergoing Colorectal Resection Inan Enhanced Recovery (Eras) Protocol: A Multicenter Randomized Clinical Trial (SONVI Study). Medicine, 95, e3704. https://doi.org/10.1097/MD.0000000000003704

[16] Mariette, C. (2015) Role of the Nutritional Support in the ERAS Programme. Journal of Visceral Surgery, 152, s18-s20. https://doi.org/10.1016/S1878-7886(15)30006-0

[17] American Society of Anesthesiologists Committee (2011) Practice Guidelines for Preoperative Fasting and the Use of Pharmacologicagents to Reduce the Risk of Pulmonary Aspiration: Application to Healthy Patients Undergoing Elective Procedures: An Updated Report by the American Society of Anesthesiologists Committee on Standards and Practice Parameters. Anesthesiology, 114, 495-511. https://doi.org/10.1097/ALN.0b013e3181fcbfd9

[18] Zhu, A.C.-C., Agarwala, A., et al. (2019) Perioperative Fluid Management in the Enhanced Recovery after Surgery (ERAS) Pathway. Clinics in Colon and Rectal Surgery, 32, 114-120. https://doi.org/10.1055/s-0038-1676476

[19] Sato, H., Carvalho, G., Sato, T., Lattermann, R., Matsukawa, T. and Schricker, T. (2010) The Association of Preoperative Glycemic Control, Intraoperative Insulin Sensitivity, and Outcomes after Cardiac Surgery. The Journal of Clinical Endocrinology \& Metabolism, 95, 4338-4344. https://doi.org/10.1210/jc.2010-0135

[20] Chen, K., Mou, Y.P., Xu, X.W., Xie, K. and Zhou, W. (2012) Necessity of Routine Nasogastric Decompression after Gastrectomy Forgastric Cancer: A Meta-Analysis. Chinese Medical Journal, 92, 1841-1844.

[21] Feldheiser, A., Aziz, O., Baldini, G., et al. (2016) Enhanced Recovery after Surgery (ERAS) for Gastrointestinal Surgery, Part 2: Consensus Statement for Anaesthesia Practice. Acta Anaesthesiologica Scandinavica, 60, 289-334. https://doi.org/10.1111/aas.12651

[22] Levy, B.F., Scott, M.J., Fawcett, W.J., Day, A. and Rockall, T.A. (2011) Optimizing Patient Outcomes in Laparoscopic Surgery. Colorectal Disease, 13, 8-11. https://doi.org/10.1111/j.1463-1318.2011.02770.x

[23] Gustafsson, U.O., Scott, M.J., Schwenk, W., et al. (2013) Guidelines for Perioperative Care in Elective Colonic Surgery: Enhanced Recovery after Surgery (ERAS) Society Recommendations. World Journal of Surgery, 37, 259-284.

https://doi.org/10.1007/s00268-012-1772-0

[24] Wang, L.H., Zhu, R.F., Gao, C., Wang, S.L. and Shen, L.Z. (2018) Application of Enhanced Recovery after Gastric Cancer Surgery: An Updated Meta-Analysis. World Journal of Gastroenterology, 24, 1562-1578. https://doi.org/10.3748/wjg.v24.i14.1562

[25] Olle, L. (2014) ERAS-Enhanced Recovery After Surgery: Moving Evidence-Based Perioperative Care to Practice. Journal of Parenteral and Enteral Nutrition, 38, 559-566.

[26] Grocott, M.P.W., Dushianthan, A., Hamilton, M.A., et al. (2013) Perioperative Increase in Global Blood Flow to Explicit Defined Goals and Outcomes after Surgery: A Cochrane Systematic Review. British Journal of Anaesthesia, 111, 535-548. https://doi.org/10.1093/bja/aet155

[27] Wu, Y.L., Cao, B., Bai, T.C., et al. (2014) Application Status and Problems of Rapid Rehabilitation Surgery in Gastric Cancer Surgery. Medical Review, 20, 643-645.

[28] Li, Z.Y., Wang, Q., Li, B.F., et al. (2017) Influence of Enhanced Recovery after Sur- 
gery Programs on Laparoscopy-Assisted Gastrectomy for Gastric Cancer: A Systematic Review and Meta-Analysis of Randomized Control Trials. World Journal of Surgical Oncology, 15, 1-9. https://doi.org/10.1186/s12957-017-1271-8

[29] Liu, H.P., Zhang, Y.C., Zhang, Y.L., Yin, L.N. and Wang, J. (2011) Drain versus No-Drain after Gastrectomy for Patients with Advanced Gastric Cancer: Systematic Review and Meta-Analysis. Digestive Surgery, 28, 178-189. https://doi.org/10.1159/000323954

[30] Simpson, J.C., Bao, M.X. and Agarwala, A. (2019) Pain Management in Enhanced Recovery after Surgery (ERAS) Protocols. Clinics in Colon and Rectal Surgery, 32, 121-128. https://doi.org/10.1055/s-0038-1676477

[31] Apfel, C.C., Turan, A., Souza, K., et al. (2013) Intravenous Acetaminophen Reduces Postoperative Nausea and Vomiting: A Systematic Review and Meta-Analysis. Pain, 154, 677-689. https://doi.org/10.1016/j.pain.2012.12.025

[32] Lee, J. and Jeon, H. (2014) The Clinical Indication and Feasibility of the Enhanced Recovery Protocol for Curative Gastric Cancer Surgery: Analysis of 147 Consecutive Experiences. Dig Sure, 31, 318-323. https://doi.org/10.1159/000368091

El Nakeeb, A., Fikry, A., El Metwally, T., et al. (2009) Early Oral Feeding in Patients Undergoing Elective Colonic Anastomosis. International Journal of Surgery, 7, 206-209. https://doi.org/10.1016/j.ijsu.2009.03.003

[33] Benjamin, R., Bell, M.D. and James, D. (2015) Prevention of Venous Thromboembolism in the Enhanced Recovery after Surgery (ERAS) Setting: An Evidence Based Review. Canadian Journal of Anesthesia, 62, 194-202. 